\title{
Analyses spectroscopiques du liquide céphalo-rachidien de rat en ex vivo et du noyau du raphé dorsal in vivo
}

\author{
S. MOTTIN, C. TRAN-MINH, P. LAPORTE*, R. CESPUGLIO** et M. JOUVET** \\ Laboratoire de Biotechnologie, Ecole Nationale Supérieure des Mines, 42023 Saint-Etienne, France \\ ${ }^{*}$ Laboratoire T.S.I., URA 842 du CNRS, Univ. J. Monnet, 42023 Saint-Etienne, France \\ ** Département de Médecine Expérimentale, INSERM U 52, Univ. Cl. Bernard, 69373 Lyon, France
}

\begin{abstract}
RÉSUMÉ
Les propriétés d'absorption et de fluorescence du liquide céphalo-rachidien (LCR) ponctionné au niveau de la cisterna magna du rat, sont analysées puis comparées à l'émission mesurée in situ dans le noyau du raphe dorsal du rat libre de tous mouvements. Les mesures de fluorescence en ex vivo du LCR et in vivo du noyau raphé dorsal, ont été réalisées par la mise en cuvre d'un microcapteur à fibre optique (FOCS). La fluorescence mesurée in vivo sous excitation à $337 \mathrm{~nm}$, présente 2 pics d'émission situés vers 410 et $460 \mathrm{~nm}$. Les spectres d'absorption, d'émission en fluorescence statique et en fluorescence induite par laser sont rapportés. Avec des domaines de longueur d'onde d'excitation de $300-315 \mathrm{~nm}, 320-355 \mathrm{~nm}$ et $360-470 \mathrm{~nm}$, les spectres d'émission du LCR en ex vivo montrent respectivement des pics centrés vers $340 \mathrm{~nm}, 390 \mathrm{~nm}$ et $530 \mathrm{~nm}$. Malgré les limites liées aux différences de localisation anatomique, ces approches ainsi que celles de la littérature permettent de suggérer que le signal de fluorescence mesuré in vivo à $460 \mathrm{~nm}$ pourrait dépendre pour une grande partie du NADH intracellulaire.
\end{abstract}

\begin{abstract}
The fluorescence properties of cerebrospinal fluid (CSF) drawn from the rat cisterna magna, are investigated and compared to the in vivo emission derived from the nucleus raphe dorsalis of the freely moving rat. Measurement in the CSF in ex vivo conditions or in situ in the nucleus raphe dorsalis, were realized by way of a fiber optic chemical sensor (FOCS). The in vivo emission, obtained with an excitation wavelength at $337 \mathrm{~nm}$, exhibits 2 peaks around 410 and $460 \mathrm{~nm}$. Absorption spectra, steady-state emission spectra and laser induced time-resolved fluorescence of CSF are reported. With excitation wavelength in the ranges $300-315 \mathrm{~nm}, 320$ $355 \mathrm{~nm}$ and $360-470 \mathrm{~nm}$, the steady-state emission spectra of CSF exhibit peaks respectively around $340 \mathrm{~nm}, 390 \mathrm{~nm}$ and $530 \mathrm{~nm}$. Despite the limitation attached to the anatomical position, the above data together with those of the literature suggest that in vivo fluorescent signal may be essentially dependent upon intracellular NADH.
\end{abstract}

\section{INTRODUCTION}

La première étude de fluorométrie réalisée in situ au niveau de la surface cérébrale, a été publiée dès 1962 par Chance, B. et coll. [1]. Plus récemment, différents montages de fibres optiques millimétriques et submillimétriques on été utilisés chez le rat anesthésié ou libre de tous mouvements pour suivre la fluorescence cérébrale du NADH [2], [3]. La nouvelle génération de FOCS implanté a pour objet la mesure des variations relatives de certains fluorophores comme le $\mathrm{NADH}$ en fonction des états de vigilance (éveil, sommeil à ondes lentes et sommeil 
paradoxal) [3]. Une des questions primordiales concernant les signaux de fluorescence dérivés du tissu cérébral, sous illumination à $337 \mathrm{~nm}$, reste le compartiment où se réalise la mesure. En effet, la pénétration du flux du laser sur des tranches cérébrales est de l'ordre de quelques centaines de microns et inférieure à $500 \mu \mathrm{m}$ [1]. Le volume analysé est donc considérablement complexe vu l'hétérogénéité du tissu cérébral. Afin d'aborder ce milieu, nous avons choisi d'explorer quelques propriétés photophysiques d'un modèle simple: le liquide céphalorachidien frâchement prélevé au niveau de la cisterna magna.

\section{II. ÉCHANTILLON ET TECHNIQUES EXPÉRIMENTALES}

Le prélèvement de LCR a été réalisé sous anesthésie au pentobarbital $(5 \mathrm{mg} / \mathrm{kg})$. Le LCR prélevé au niveau de la cisterna magna se présente sous la forme de liquide incolore légèrement opalescent. Tout échantillon présentant des traces de sang a été écarté. Chaque échantillon analysé, de l'ordre de $200 \mu \mathrm{l}$, provient du prélèvement de deux rats males OFA/CREDO.

Les mesures d'absorption ont été réalisées sur un modèle 860 Uvikon de la société Kontron. La fluorescence statique a été mesurée sur un modèle F4500 de la société Hitachi.

La technique instrumentale et le capteur utilisés pour les mesures in vivo ont été décrits précédemment [3], [4]. Nous présentons le montage succintement (Figure 1). Le capteur monofibre et monobrin de $200 \mu \mathrm{m}$ de diamètre, met en œuvre des longueurs de quelques mètres.

Le monochromateur est utilisé avec une résolution de $4 \mathrm{~nm}$.

Le laser fonctionne à $20 \mathrm{~Hz}$. L'impulsion du laser azote (longueur d'onde de 337nm) est de 300 ps à mi-hauteur. L'impulsion de fluorescence est intégrée sur une fenêtre temporelle de 10ns.

Les aires intégrées sont moyennées sur 256 coups lasers.

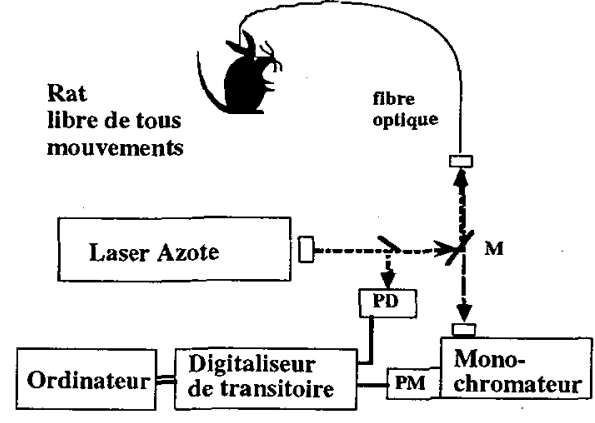

Figure 1: Représentation du montage expérimental utilisé in vivo. PD: photodiode; PM: photomultiplicateur; M: mirroir dichroïque.

\section{RÉSULTATS EXPÉRIMENTAUX ET DISCUSSION}

La Figure 2 présente quatre spectres non corrigés d'émissions typiques de fluorescence obtenus en fonction du temps in vivo au niveau du noyau du raphe dorsal. Le spectre d'émission lié au bruit intrinsèque du capteur à fibre optique est indiqué. Sur les spectres in vivo il apparaît clairement deux pics d'émission centré vers $400 \mathrm{~nm}$ et $460 \mathrm{~nm}$. Le pic mesuré à $460 \mathrm{~nm}$ est attribué majoritairement au NADH présent dans le volume exploré [1].

Le spectre d'émission d'une solution de NADH est mesuré dans les mêmes conditions juste après ces mesures in vivo. Cette mesure in vitro permet la comparaison avec les signaux d'émission mesurés in vivo.

Afin de tenter d'élucider de quel compartiment provient la mesure des 2 pics d'émission observés in vivo, nous avons entrepris 3 types de mesures :

- absorption du LCR dans la gamme 240-340 nm;

- fluorescence statique du LCR pour des longueurs d'onde d'émission de 300 à $600 \mathrm{~nm}$ et d'excitation de 300 à 400 nm; 
- fluorescence du LCR induite par laser et mesurée dans des conditions comparables à celles in vivo.

Sur la Figure 3, le spectre d'absorption moyen du LCR dilué et non dilué ( $\mathrm{n}=5$ ), présente un pic centré vers $266 \mathrm{~nm}$. Sur du LCR non dilué, la transmission de la lumière à $266 \mathrm{~nm}$ est de seulement $10 \%$ au bout de $33 \mu \mathrm{m}$. Il faut souligner l'absence de pic d'absorption mesurable à $340 \mathrm{~nm}$. La densité optique mesurée à $337 \mathrm{~nm}$ est d'environ 0.02 , soit $95 \%$ de transmission au bout de $200 \mu \mathrm{m}$.

Avec des domaines de longueur d'onde d'excitation de $300-315 \mathrm{~nm}, 320-355 \mathrm{~nm}$ et $360-470$ $\mathrm{nm}$, les spectres d'émission du LCR en ex vivo montrent respectivement des pics centrés vers $340 \mathrm{~nm}, 390 \mathrm{~nm}$ et $530 \mathrm{~nm}$ (Figure 4). L'émission vers $340 \mathrm{~nm}$ est largement plus importante que les deux autres. Pour des longueurs d'onde d'excitation inférieures à $315 \mathrm{~nm}$, seule l'émission centrée à $340 \mathrm{~nm}$ a pu être observée avec un maximum d'émission pour des excitations à 230 et $295 \mathrm{~nm}$. La même émission est retrouvée dans le sérum humain [5]. L'émission à $340 \mathrm{~nm}$ peut être raisonnablement attribuée au tryptophane bien que celle-ci s'étende un peu plus vers le rouge. En outre, certains auteurs ont rapporté que d'autres composés que le tryptophane présentaient des données spectrales assez semblables dans la région 295/405nm (longueur d'onde d'excitation/longueur d'onde d'émission) dans le sérum [6].

Pour des longueurs d'onde d'excitation supérieures à $315 \mathrm{~nm}$, de faibles émissions ont été identifiées: l'une est centrée vers $390 \mathrm{~nm}$, l'autre vers $530 \mathrm{~nm}$. L'attribution moléculaire de l'émission à $390 \mathrm{~nm}$ n'est pas aisée. Certains auteurs ont détecté la présence de nombreux composés endogènes fluorescents dans l'ultrafiltrat de sérum humain par HPLC, avec une détection à $415 \mathrm{~nm}$ et une excitation à $322 \mathrm{~nm}$ [7]. Concernant les dérivés du tryptophane, Wolfbeis et coll. ont présenté certaines identifications moléculaires [5], [8] : l'indoxyl sulfate (indican) 290/385 nm, l'acide 3-Hydroxy-anthranilique 320/415 nm, et les composés de la famille de la vitamine $B_{6}$ (pyridoxine) 290-320/390 nm. Ces résultats sont retrouvés pour le sérum humain [5]. Wolfbeis et coll. attribuent les pics à $450 / 530 \mathrm{~nm}$ à la bilirubine liée à l'albumine. Les pics vers $370 / 530 \mathrm{~nm}$ sont, eux, partiellement identifiés et proviendraient des flavines (riboflavine, vitamine $B_{2} \ldots$ ) [5]. Contrairement aux résultats obtenus avec le sérum [5], aucune émission attribuable au NADH n'a été détectée à 460nm.

Nous avons reporté sur la Figure 5 les spectres d'émission du LCR mesurés avec le FOCS (longueur d'onde d'excitation égale à $337 \mathrm{~nm}$ ). En accord avec les résultats obtenus en absorption et en fluorescence statique, seule une très faible fluorescence (vers $450-460 \mathrm{~nm}$ ) a pu être mesurée dans ces conditions.

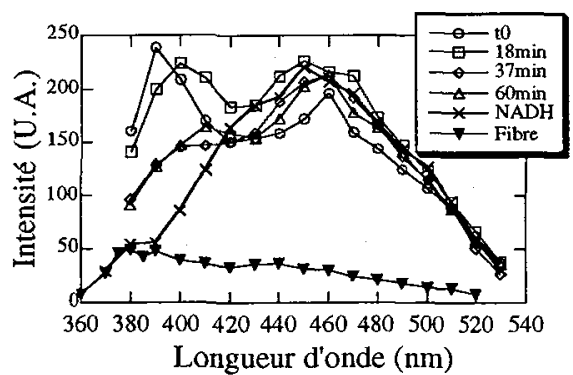

Figure 2: Spectres d'émission des noyaux du raphe dorsal obtenus in vivo (symboles ouverts), d'une solution de NADH (pH7, concentration égale à $15 \mu \mathrm{M})$ et de la fibre plongeant dans de l'eau. Le temps indique correspond au premier point du spectre $(380 \mathrm{~nm})$.

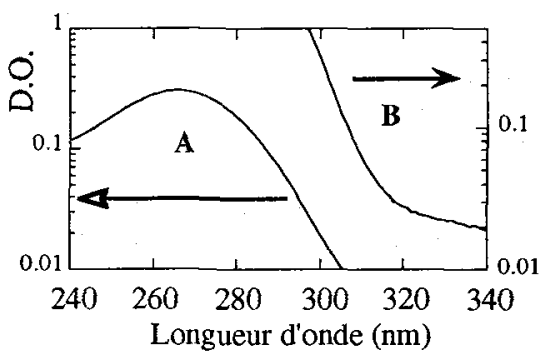

Figure 3: Spectre d'absorption du LCR (trajet d'absorption égal à $200 \mu \mathrm{m}$ ). Les courbes A et B correspondent respectivement aux spectres du LCR dilué à $1 / 20$ et sans dilution. 


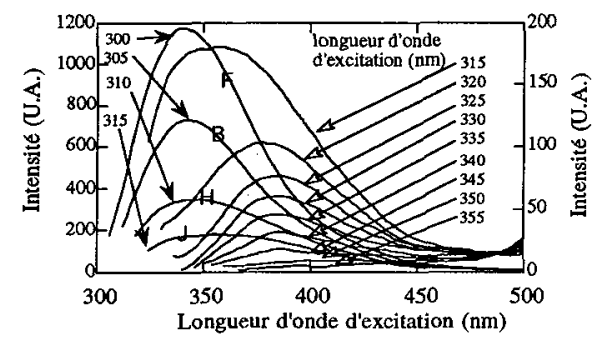

Figure 4: Spectres d'émission du LCR à différentes longueurs d'ondes d'excitation obtenus en fluorescence statique. Les courbes avec des symboles ont leurs ordonnés à gauche.

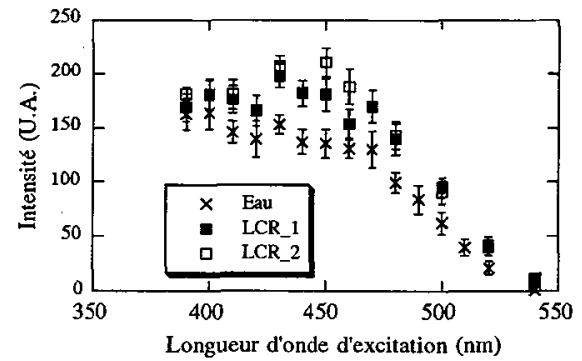

Figure 5: Spectres d'émission du LCR mesurés par le capteur à fibre optique sous excitation laser à $337 \mathrm{~nm}$. Chacune des courbes LCR_1 et LCR_2 proviennent d'un echantillon issu d'un prélèvement sur 2 rats. Les barres correspondent a la déviation standard de 5 mesures successives.

\section{CONCLUSION}

A la vue de ces résultats, il pourrait être suggéré que les mesures réalisées dans le tissu soient peu affectées par le LCR où elles baignent. Il faut toutefois marquer une réserve car les LCR respectivement des cavités et du tissu peuvent être différents. Ce dernier peut refléter des évènements locaux variables et parfois opposés selon la zone cérébrale considérée; le LCR ventriculaire ne reflète que l'intégrale de ces variations spécifiques. Pour prendre en compte ces réserves, le terme de fluorophores "endogènes" englobant les compartiments intracellulaire et extracellulaire est le plus approprié. Ainsi l'émission de fluorescence mesurée in vivo à $460 \mathrm{~nm}$ par le FOCS au niveau du noyau raphé dorsal et liée au NADH endogène, pourrait provenir du compartiment intracellulaire essentiellement. L'étude des variations du NADH endogène et des fluorophores responsables de l'émission à $400 \mathrm{~nm}$ en fonction des états de vigilance est en cours.

\section{RÉFÉRENCES}

[1] B. Chance, P. Cohen, F. Jobsis et B. Schoener, Science, 1962, 137, pp. 499-508.

[2] A. Mayevsky and B. Chance, Science, 1982, 217, pp. 537-540.

[3] S. Mottin, C. Tran-Minh, P. Laporte, R. Cespuglio et M. Jouvet, IEEE Engineering in medecine and biology society proc. $14^{\text {th }}$ Annual Int. Conf. 1992, pp. 235-238.

[4] S. Mottin, C. Tran-Minh, P. Laporte, R. Cespuglio et M. Jouvet, Applied Spectroscopy, 1993, 47, pp. 590-597.

[5] O. S. Wolfbeis et M. Leiner, Anal.Chim.Acta; 1985, 167, pp. 203-215.

[6] J. S. Swan, E. Y. Kragten et H. Veening, Clin.Chem; 1983, 29, 6, pp. 1082-1084.

[7] H. Mabuchi, H. Nakahashi, Clin.Chem; 1983, 29, 4, pp. 675-677.

[8] O.S. Wolfbeis, S. G. Schulman, The fluorescenec of organic natural products; Molecular Luminescence Spectroscopy, method and applications: Part 1; New-York; Wiley J. \& sons; 1985 , 77, pp. $167-370$. 